\title{
Role of coronary CT angiography in anomalous high take-off of right coronary artery with ST elevation myocardial infarction: a case report
}

\author{
Hyun Kuk Kim, Sung Soo Kim, Young Jae Ki, Dong Hyun Choi, Keun Ho Park \\ Department of Cardiovascular Medicine, Chosun University Medical School, Gwangju, Korea
}

Received January 4, 2021

Revised January 14, 2021

Accepted January 15, 2021

Corresponding author

Sung Soo Kim

Department of Cardiovascular

Medicine, Chosun University

Medical School, 365 Pilmundaero,

Dong-gu, Gwangju 61452, Korea

Tel: $+82-62-220-3240$

Fax: +82-62-228-7174

E-mail: kholywater@gmail.com

ORCID:

http://orcid.org/0000-0002-5190-227X

\begin{abstract}
Anomalous high take-off of the right coronary artery (RCA) ostium above the sinuses of valsalva is extremely rare. It was not well visualized on conventional coronary angiography. A 52-year-old man was referred to emergency department presenting as chest pain. Because electrocardiography showed ST elevation in the inferior lead, he underwent emergent cardiac catherization, which revealed mild stenosis in the left coronary artery. However, the ostium of the RCA could not be found arising from the coronary cusp despite using aortogram with pigtail. Intraprocedural coronary computed tomography angiography showed a near total occlusion in the middle RCA, which had an anomalous take-off from the left anterior superior aspect of the ascending aorta, $2 \mathrm{~cm}$ above the left main coronary artery. Judkins left 4 guiding catheter via femoral approach could be engaged in the ostium of RCA. Successful percutaneous angioplasty using the Guidezilla extension catheter as additional backup support was performed.
\end{abstract}

Keywords: Anomaly; Coronary vessel; Multidetector computed tomography; Myocardial infarction

\section{INTRODUCTION}

Anomalous coronary artery arising from the opposite sinus is a rare phenomenon, and its estimated total prevalence rate was found to be $1.07 \%$ [1]. Among them, a high take-off right coronary artery (RCA) ostium above the sinuses of Valsalva is extremely rare [2,3]. Because of the unusual anatomy, cases of high take-off RCA were easily misdiagnosed or not well visualized on conventional coronary angiography. In this situation, intraprocedural coronary computed tomography angiography (CCTA) is a useful imaging modality in the emergency department reducing total ischemic time in patients with ST elevation myocardial infarction (STEMI)
[4]. We report a rare case of an anomalous high take-off RCA presenting with STEMI, which was successfully treated with the help of intraprocedural CCTA.

Written informed consent was obtained from the patient for publication of this case report, which was approved by the Institutional Review Board of the Chosun University Hospital (CHOSUN 2020-11-028).

\section{CASE REPORT}

A 52-year-old man was referred to our emergency department complaining of squeezing chest discomfort that had increased over the past 6 hours. He had diabetes mellitus 
and had smoked around 10 cigarettes daily for the past 30 years. On admission, his blood pressure was 126/80 mmHg, and his pulse was 84 beats/minute and regular. Twelve-lead electrocardiography showed ST segment elevation in the II, III, and aVF leads, which suggested that the infarct-related artery was the RCA (Fig. 1). The serum creatine kinase - MB fraction was $78.4 \mathrm{ng} / \mathrm{mL}$ (normal range $0-4.88$ ) and troponin - I was $3.69 \mathrm{ng} / \mathrm{mL}$ (normal range 0-0.016).

He underwent emergency cardiac catheterization, which revealed mild stenosis in the left anterior descending coronary artery and the left circumflex artery (Fig. 2A). However, the ostium of the RCA could not be found via routine diagnostic catheter arising from the coronary cusp despite using aortogram with pigtail catheters (Royal Flush Plus, Bloomington, IN, USA) (Fig. 2B). Several attempts at relieving the symptoms failed, and the patient still complained of squeezing pain. Finally, we decided to check an intraproce- dural CCTA, which was taken after moving the patient from the catheterization laboratory to the computed tomography room while maintaining the arterial sheath. CCTA was performed. A 320 slice CT scanner (Aquilion ONE, Toshiba Medical Systems, Nasu, Japan) with three-dimensional volumetric cardiac imaging during the diastole of $\mathrm{R}-\mathrm{R}$ interval (Gantry rotation time of $350 \mathrm{msec}$, scan time of $1.224 \mathrm{sec}, 0.5$ mm slice thickness with 0.25 reconstruction interval) was done and chamber views, curved multiplanar reformation (MPR) images, maximum intensity projection (MIP) images, and volume rendering images were acquired. In CCTA, $80 \mathrm{~mL}$ of contrast agent, Tomoray 320 (Ioversol; Dongguk Pharm, Seoul, Korea), followed by $40 \mathrm{~mL}$ of a $50 \% / 50 \%$ saline/contrast medium mixture was infused at $4.5 \mathrm{~mL} / \mathrm{sec}$ by a dual injection system (Stellant, Medrad Inc., Indianola, PA, USA). CCTA showed a near total occlusion in the middle RCA, which had an anomalous take-off from the left ante-
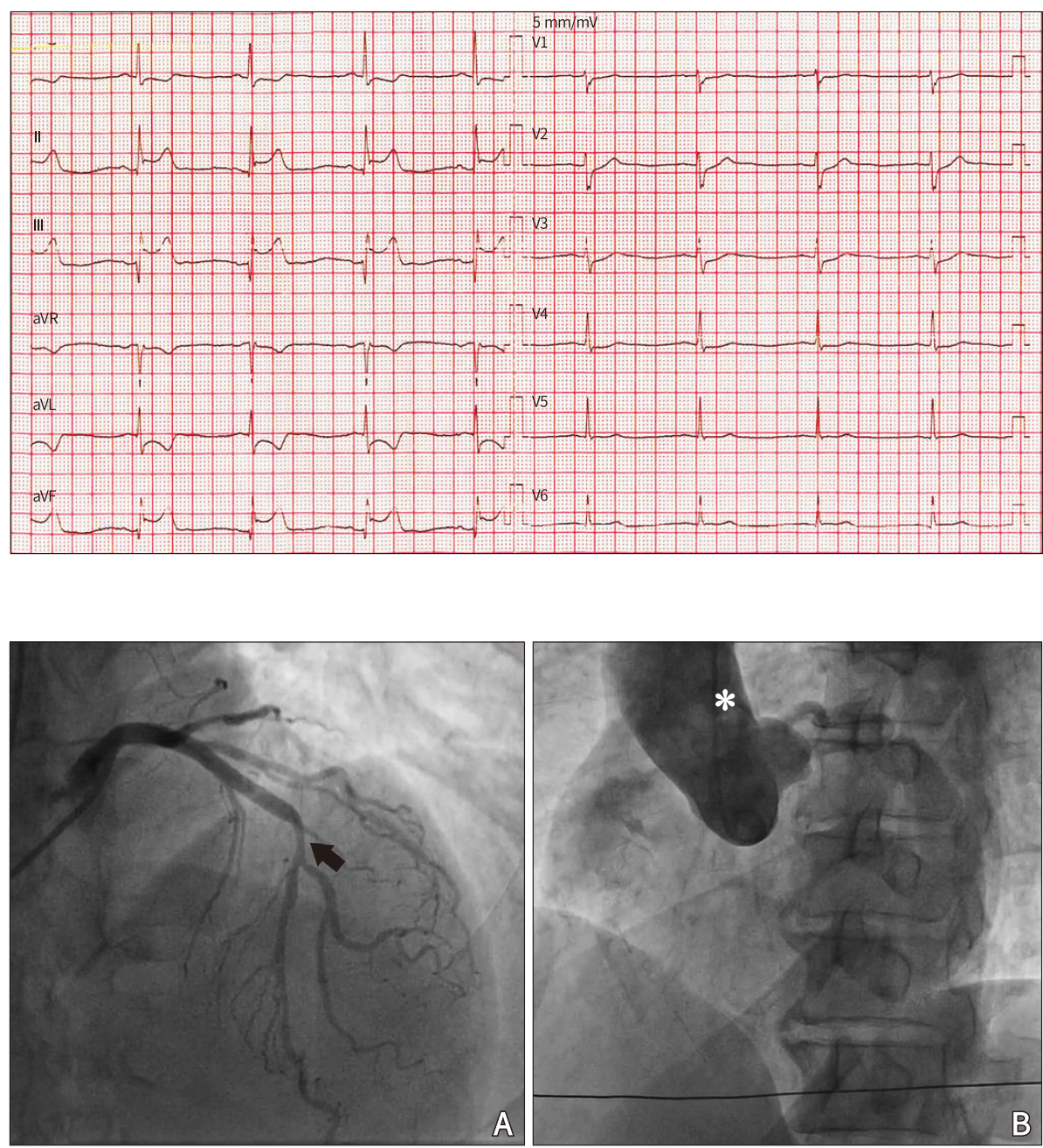

Fig. 1. Twelve-lead electrocardiography. Twelvelead electrocardiography showed ST segment elevation in the II, III, and aVF leads, which suggested that the infarct-related artery was the right coronary artery. 
rior superior aspect of the ascending aorta, $2 \mathrm{~cm}$ above the origin of the left main coronary artery (Fig. 3A). It then shifted leftward and coursed between the pulmonary artery and the aorta (Fig. 3B).

The patient returned to the cathroom and underwent cardiac catheterization again. A 6 Fr Judkins left 4 guiding catheter (Cordis, Miami, FL, USA) via a femoral approach was engaged in the RCA ostium (Fig. 4A). After a $2.5 \mathrm{~mm}$ semi compliant balloon inflation, we deployed three zotalimus-eluting stents $(2.75 \times 38 \mathrm{~mm}, 2.75 \times 40$ $\mathrm{mm}$ and $3.0 \times 18 \mathrm{~mm}$ Resolute Onyx ${ }^{\mathrm{TM}}$, Medtronic Cardiovascular, Santa Rosa, CA, USA) in the middle and distal RCA (Fig. 4B) with a Guidezilla ${ }^{\mathrm{TM}}$ guide extension catheter (Boston Scientific, Marlborough, MA, USA). A final Angiography demonstrated favorable dilation of the target lesion. Total procedure time was about 180 minutes. The patient tolerated the entire procedure and we success- fully completed the treatment. He followed an uneventful hospital course and was, consequently, discharged. The patient quit smoking and was prescribed dual antiplatelet agent, beta blocker and statin. He has remained asymptomatic during for over 3 year. A follow up two-dimensional echocardiography showed that the ejection fraction had recovered to $>50 \%$.

\section{DISCUSSION}

Since the benefits of reperfusion decline rapidly with time, prompt restoration of blood flow in the infarct-related coronary artery is important for reducing mortality and myocardial salvage in patients with STEMI [5,6]. However, unusual locations and courses of coronary artery anomalies pose considerable challenges to cardiologists. In particular, a high take-off RCA is a very rare finding, easily misdiag-

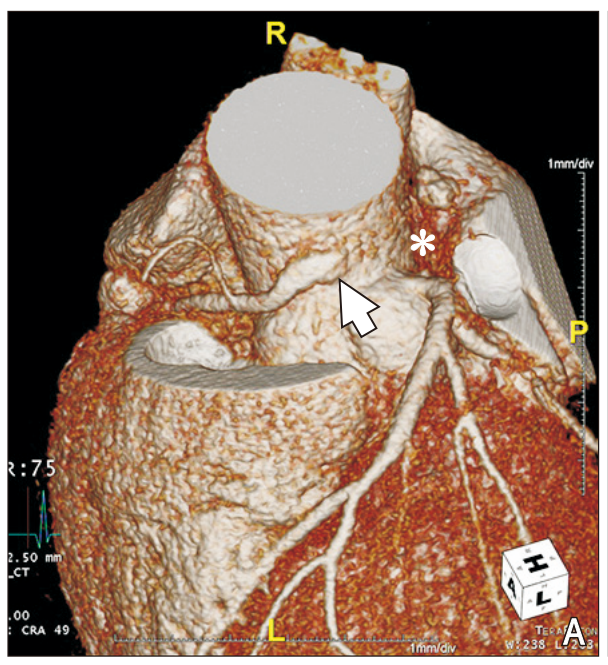

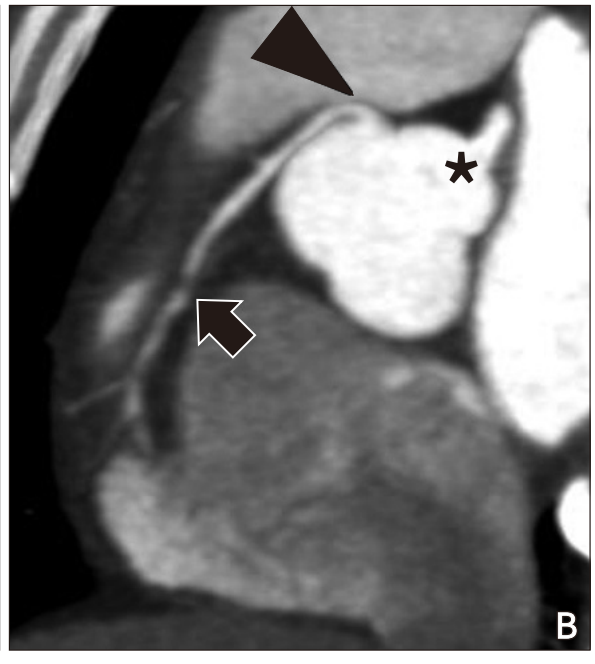

B
Fig. 3. Coronary computed tomography angiography. (A) Volume rendering image shows near total occlusion in the mid portion of right coronary artery, which had an anomalous take-off from the left anterior superior aspect of ascending aorta (solid arrow head), 2 $\mathrm{cm}$ above the origin of left main coronary artery (asterisk). It then shifted leftward and coursed between the pulmonary artery and the aorta. (B) Maximum intensity projection image represents an anomalous origin of right coronary artery, which originated from the aorta $2 \mathrm{~cm}$ above the sinotubular junction. Solid arrow indicates the ostium of the right coronary artery, asterisk indicates the ostium of the left main coronary artery and black arrow indicates the target lesion.
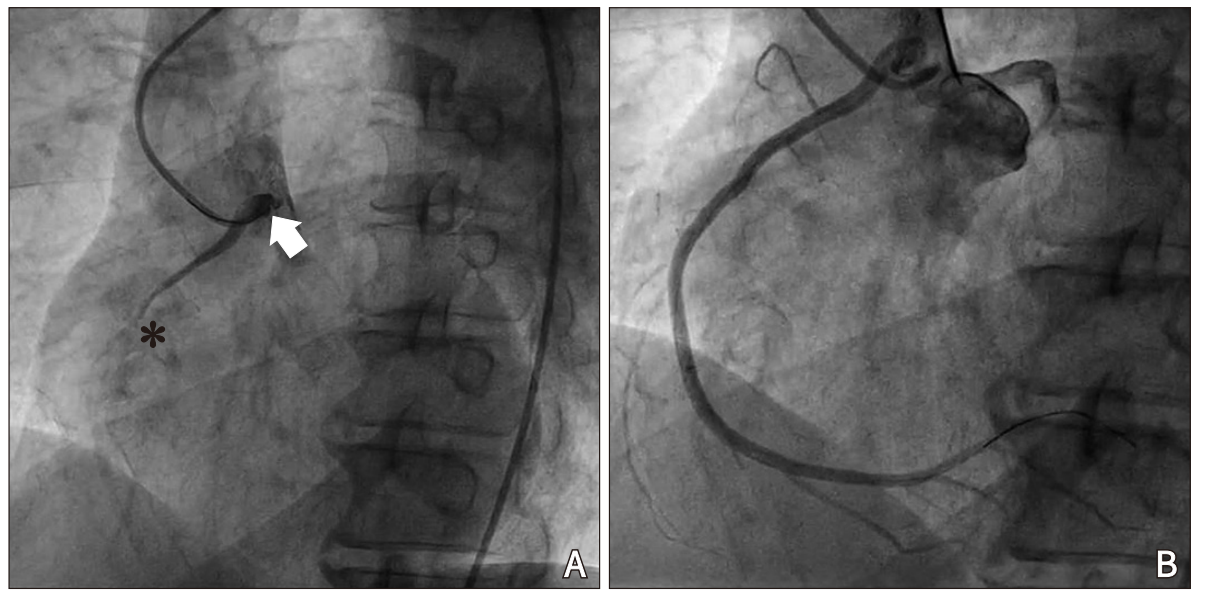

Fig. 4. Percutaneous coronary Intervention. (A) Coronary angiography showed a total occlusive lesion in the mid portion of right coronary artery, left anterior oblique view. Solid arrow indicates the ostium of the right coronary artery, asterisk indicates target lesion. (B) Final coronary angiography showed favorable dilation of the target lesion. 
nosed or poorly visualized by coronary angiography. Conventional techniques and catheters might fail to find and anchor into the RCA's orifice, and restoration of blood flow could be delayed.

In our case, the ostium of RCA could not be found despite using aortogram with pigtail catheters; so, we decided to check the intraprocedural CCTA. CCTA is a noninvasive imaging technique that provides an excellent overview of the complex cardiac vascular anatomy and establishes a capacity to view the heart in the necessary three-dimensional image to precisely identify coronary pathways and other potential anomalous structures [7]. This helped us valuable data in demonstrating the high aortocoronary take-off the RCA and its spatial relationships with the heart and great vessels.

Because of the unusual anatomy of an anomalous origin of the RCA from the left sinus of Valsalva, selective cannulation of the guiding catheter for cases requiring PCI is always challenging. According to one study, a FL (forward takeoff Judkins) 3.0 catheter was recommended for anomalous RCA above the left sinotubular plane [8]. The FL catheter has a secondary radius or curvature, slightly smaller than a Judkin left catheter, which allows to act as a hook and engage an RCA originating above the left coronary artery at an angle and then coursing inferiorly and to the right. Firstly, we use the Judkin Right 4, Amplatz Left 1 guiding because the FL catheter was not available in our hospital. However, these guiding catheters were floating in the ascending aorta and neither was not successful. Subsequently, we used the Judkin left 4 guiding catheter, which could be closest to the ostium of the RCA in the ascending aorta. For anomalous RCA originating above the left sinus of Valsalva just adjacent to the LCA, the JL catheter with a larger secondary curve than the one used to cannulate the left coronary ostium was successful in providing engagement for anomalous RCA. The Judkins left catheter was pushed deep into the left sinus of Valsalva causing it to make an anterior and cephalad pointing $\mathrm{U}$ turn. This larger curve prevented the catheter from automatically engaging the left coronary and allowed for selective cannulation of the anomalous RCA without difficulty [9]. Even though this JL guiding catheter was inadequate alignment with the initial course of RCA in our case, the Guidezilla guide extension catheter as additional backup support for the guiding catheter was used, and suc- cessfully deployed these stents in this complex case of PCI. Also, switching the right radial approach or other guiding catheter such as Amplatz Left 2, Multipurpose, Left coronary bypass catheter might be the another good option in these cases [10].

Although coronary anomaly such as high take-off of RCA is often detected in CCTA, high take-off of RCA in patient with STEMI needs urgent accurate diagnosis and more attention. CCTA with volume rendering and other planar images including MIP images may render useful informations to cardiologists for localization of coronary anomalies and planning treatment, reducing total ischemic time in patients with STEMI.

\section{ACKNOWLEDGEMENTS}

This study was supported by research funds from Chosun University Hospital, 2020.

\section{CONFLICT OF INTEREST}

No potential conflict of interest relevant to this article was reported.

\section{REFERENCES}

1. Kragel AH, Roberts WC. Anomalous origin of either the right or left main coronary artery from the aorta with subsequent coursing between aorta and pulmonary trunk: analysis of 32 necropsy cases. Am J Cardiol 1988;62:771-7.

2. Yamanaka O, Hobbs RE. Coronary artery anomalies in 126,595 patients undergoing coronary arteriography. Cathet Cardiovasc Diagn 1990;21:28-40.

3. Thakur R, Dwivedi SK, Puri VK. Unusual "high take off" of the right coronary artery from the ascending aorta. Int J Cardiol 1990;26:369-71.

4. Agarwal PP, Dennie C, Pena E, Nguyen E, LaBounty T, Yang $\mathrm{B}$, et al. Anomalous coronary arteries that need intervention: review of pre- and postoperative imaging appearances. Radiographics 2017;37:740-57.

5. Hampton J, Wilcox R, Armstrong P, Aylward P, Bett N, Charbonnier B, et al. Late Assessment of Thrombolytic Efficacy (LATE) study with alteplase 6-24 hours after onset of acute myocardial infarction. Lancet 1993;342:759-66.

6. Ibanez B, James S, Agewall S, Antunes MJ, Bucciarelli-Ducci C, Bueno H, et al. 2017 ESC guidelines for the management 
of acute myocardial infarction in patients presenting with ST-segment elevation: the task force for the management of acute myocardial infarction in patients presenting with ST-segment elevation of the European Society of Cardiology (ESC). Eur Heart J 2018;39:119-77.

7. Kim BK, Cho I, Hong MK, Chang HJ, Shin DH, Kim JS, et al. Usefulness of intraprocedural coronary computed tomographic angiography during intervention for chronic total coronary occlusion. Am J Cardiol 2016;117:1868-76.

8. Sarkar K, Sharma SK, Kini AS. Catheter selection for coronary angiography and intervention in anomalous right coro- nary arteries. J Interv Cardiol 2009;22:234-9.

9. Uthayakumaran K, Subban V, Lakshmanan A, Pakshirajan B, Solirajaram R, Krishnamoorthy J, et al. Coronary intervention in anomalous origin of the right coronary artery (ARCA) from the left sinus of valsalva (LSOV): a single center experience. Indian Heart J 2014;66:430-4.

10. Abbasov E, Bagirov I, Akhundova A. Radial approach is better than the femoral one in anomalous high RCA take-off from the left-anterior part of the ascending aorta. J Cardiol Cases 2013;7:e126-8. 